\title{
DABCO-Catalyzed Green Synthesis of 2-Hydroxy-1,4-diones via Direct Aldol Reaction of Arylglyoxals in Water
}

\author{
Mahnaz Saraei*, Bagher Eftekhari-Sis ${ }^{\dagger, *}$, and Sakineh Mozaffarnia ${ }^{\dagger}$ \\ Department of Chemistry, Payame Noor University, P. O. Box. 19395-3697, Tehran, Iran. \\ ${ }^{*}$ E-mail: m_saraii@yahoo.com \\ 'Department of Chemistry, University of Maragheh, P. O. Box. 55181-83111, Maragheh, Iran. \\ *E-mail: eftekharisis@maragheh.ac.ir \\ (Received November 27, 2012; Accepted March 13, 2013)
}

\begin{abstract}
A green and simple method to synthesize of 1,4-diketones via aldol reaction of arylglyoxals and ketones such as 1-(4-methoxyphenyl)-2-propanone, deoxybenzoin and substituted acetophenones in the presence of a catalytic amount of $\mathrm{DABCO}$ in water at room temperature has been reported. Corresponding 2-hydroxy-1,4-diones were obtained in moderate to high yields with simple separation of obtained solid from reaction mixture and recrystallization.
\end{abstract}

Key words: 1,4-Diketones, Aldol reaction, Arylglyoxals, DABCO, Green chemistry

\section{INTRODUCTION}

Because of water is one of the most abundant and cheap solvents, and also makes the reactions environmentally amenable, much safer and easier to handle, ${ }^{1}$ the organic reactions in aqueous media have attracted much attention in synthetic organic chemistry. ${ }^{2}$ Also, reactions in water exhibit interesting reactivity and selectivity, which are different from conventional organic solvents.

The routes for formation of $\mathrm{C}-\mathrm{C}$ bonds are the most important topics in synthetic organic chemistry. The aldol reaction is one of the most powerful $\mathrm{C}-\mathrm{C}$ bond-forming reactions in organic synthesis, ${ }^{3}$ leading to the $\beta$-hydroxycarbonyl structural units, which are found in several biologically active compounds, such as macrolide antibiotics and anti-cancer drugs, ${ }^{4}$ and also are important building blocks in the synthesis of polyfunctional compounds and natural products. ${ }^{3 \mathrm{~d}, 5}$

Additionally, 1,4-dicarbonyl compounds are versatile precursors for the synthesis of substituted cyclopentenones, ${ }^{6}$ such as jasmones, ${ }^{6 \mathrm{~g}-\mathrm{i}}$ cuparenones,${ }^{6 \mathrm{c}}$ and prostaglandins, ${ }^{6 j-1}$ and also various heterocyclic compounds, for example, furan, thiophene, pyrrole, and pyridazine derivatives. ${ }^{7}$ Thus the development of the synthetic methods of the 1,4-diketones have a significant impact on organic chemistry. A number of methods have already been reported for the preparation of 1,4-diketone derivatives, ${ }^{8}$ and among them, most widely used approach is the Michael addition to $\alpha, \beta$-unsaturated ketones of either unmasked acyl anions such as acyllithium, ${ }^{9}$ and acyl-transition metal complexes, ${ }^{10}$ or masked acyl anions and their equivalents. ${ }^{11}$ Also, the preparation of 1,4-dicarbonyl compounds were carried out by the classic utilization of $\alpha$-halo ketones, ${ }^{12}$ by application of the Stetter reagent, ${ }^{13}$ thiazolium salts in the presence of a tertiary amine, ${ }^{14}$ or 1,3-dithiane derivatives. ${ }^{15}$

Recently, we have reviewed the application of arylglyoxals (aromatic $\alpha$-ketoaldehyds) in synthesis of heterocyclic compounds, ${ }^{16}$ that encouraged us to work on aldol reaction of arylglyoxals to prepare 1,4-dicarbonyl compounds.

However, aldol reaction with some $\alpha$-dicarbonyl compounds such as benzil, isatin and ethyl phenylglyoxalate ${ }^{17}$ to synthesize of 1,4-dicarbonyl compounds were previously reported, ${ }^{18}$ as the best of our knowledge, there is one report on aldol reaction of arylglyoxals in the literature using expensive chiral ligand with limited scope of products, in which trimethylsilyl enols of ketones were used in $\mathrm{CH}_{2} \mathrm{Cl}_{2}{ }^{19}$

Herein, we wish to report the direct aldol reaction of different ketones such as 1-(4-methoxyphenyl)-2-propanone, deoxybenzoin and substituted acetophenones with arylglyoxals in water to produce 2-hydroxy-1,4-diketones in the presence of a catalytic amount of DABCO.

\section{RESULTS AND DISCUSSION}

We first studied the aldol reaction of 1-(4-methoxyphenyl)propan-2-one 1a with phenylglyoxal hydrate $\mathbf{2 a}$, which were prepared by oxidation of acetophenone using $\mathrm{SeO}_{2},{ }^{20}$ in water in the presence of different tertiary amines such 


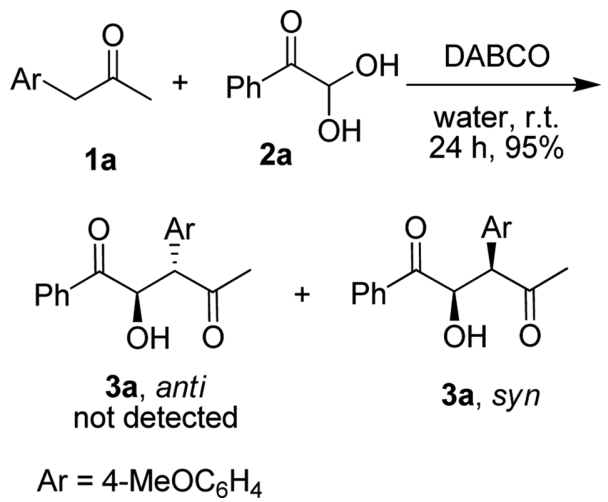

Scheme 1. Reaction of 1a with phenylglyoxal hydrate $2 \mathrm{a}$ in water.

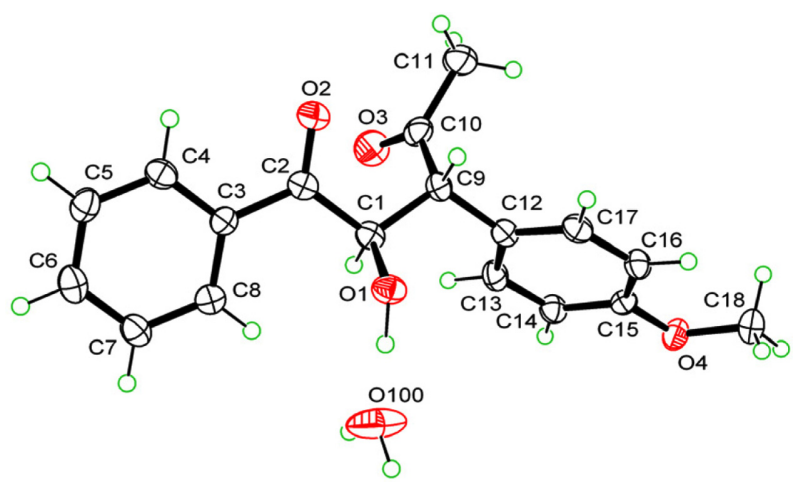

Fig. 1. ORTEP representation of $\mathbf{3 a}$ with $45 \%$ ellipsoid probability. ${ }^{21}$

as $\mathrm{Et}_{3} \mathrm{~N}$, DMAP and $\mathrm{DABCO}$, and also $\mathrm{NaOH}$ at room temperature to give 2-hydroxy-3-(4-methoxyphenyl)-1phenylpentane-1,4-dione 3a (Scheme 1). When DABCO was used, 2-hydroxy-1,4-dione 3a was obtained in 95\% yield as only syn isomer, determined using ${ }^{1} \mathrm{H}$ NMR spectroscopy. The crystal structure of the compound 3a (Fig. $1),{ }^{21}$ which was synthesized by the similar procedure, ${ }^{22}$ was reported by K. Harms, ${ }^{23}$ which is consistent with $s y n$ isomer.

Aldol reactions of $\mathbf{1 a}$ with different substituted phenylglyoxal hydrates $\mathbf{2} \mathbf{b}-\mathbf{f}$ were also investigated. The reactions were carried out by addition of DABCO to a mixture of $1 \mathrm{a}(0.5 \mathrm{mmol})$ and $2(0.05 \mathrm{mmol})$ in water and stirred at room temperature for appropriate time. The corresponding 2-hydroxy-1,4-diones $\mathbf{3 b}-\mathbf{f}$ were obtained in good to high yields. Also the aldol reaction of deoxybenzoin $\mathbf{1 b}$ was conducted with different arylglyoxals $\mathbf{2}$ under the same conditions, and the corresponding 2-hydroxy1,4-diones 3g-i were obtained in good to high yields (Scheme 2). The results are summarized in Table 1. The products were characterized using FT-IR, ${ }^{1} \mathrm{H}$ NMR and ${ }^{13} \mathrm{C}$ NMR spectroscopy.

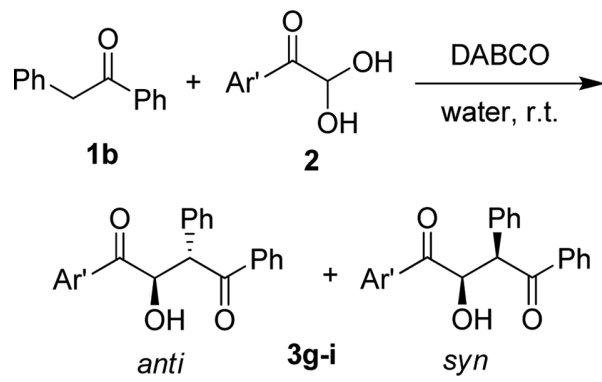

Scheme 2. Reaction of $\mathbf{1 b}$ with arylglyoxal hydrates in water in the presence of DABCO.

Table 1. Aldol reaction of $\mathbf{1 a}$ and $\mathbf{1 b}$ with arylglyoxal hydrates 2 in the presence of DABCO in water. ${ }^{a}$

\begin{tabular}{ccccc}
\hline $\begin{array}{c}\text { Ketone } \\
{[1]}\end{array}$ & $\operatorname{Ar}^{\prime}[2]$ & $\begin{array}{c}\text { Product } \\
{[3]}\end{array}$ & $\begin{array}{c}\text { Yield } \\
(\%)^{b}\end{array}$ & syn/antic \\
\hline $\mathbf{1 a}$ & $\mathrm{Ph}[\mathbf{2 a}]$ & $\mathbf{3 a}$ & 95 & $>99 / 1$ \\
$\mathbf{1 a}$ & $4-\mathrm{ClC}_{6} \mathrm{H}_{4}[\mathbf{2 b}]$ & $\mathbf{3 b}$ & 77 & $>99 / 1$ \\
$\mathbf{1 a}$ & $4-\mathrm{BrC}_{6} \mathrm{H}_{4}[\mathbf{2 c}]$ & $\mathbf{3 c}$ & 84 & $>99 / 1$ \\
$\mathbf{1 a}$ & $4-\mathrm{MeOC}_{6} \mathrm{H}_{4}[\mathbf{2 d}]$ & $\mathbf{3 d}$ & 82 & $67 / 33$ \\
$\mathbf{1 a}$ & $3-\mathrm{MeOC}_{6} \mathrm{H}_{4}[\mathbf{2 e}]$ & $\mathbf{3 e}$ & 74 & $67 / 33$ \\
$\mathbf{1 a}$ & $3,4-\left(\mathrm{MeO}_{2} \mathrm{C}_{6} \mathrm{H}_{3}[\mathbf{2 f}]\right.$ & $\mathbf{3 f}$ & 72 & $64 / 36$ \\
$\mathbf{1 b}$ & $4-\mathrm{ClC}_{6} \mathrm{H}_{4}[\mathbf{2 b}]$ & $\mathbf{3 g}$ & 70 & $>99 / 1$ \\
$\mathbf{1 b}$ & $4-\mathrm{BrC}_{6} \mathrm{H}_{4}[\mathbf{2 c}]$ & $\mathbf{3 h}$ & 82 & $>99 / 1$ \\
$\mathbf{1 b}$ & $4-\mathrm{MeOC}_{6} \mathrm{H}_{4}[\mathbf{2 d}]$ & $\mathbf{3 i}$ & 80 & $65 / 35$ \\
\hline
\end{tabular}

${ }^{a}$ Reaction time was $24 \mathrm{~h}$, except in the case of $3 \mathbf{i}$ which was $48 \mathrm{~h}$.

${ }^{b}$ Yields refer to isolated products by simple filtration of the reaction mixture.

${ }^{c}$ Anti/syn ratio was determined using ${ }^{1} \mathrm{H}$ NMR spectroscopic analysis of the crude reaction mixture.

As shown in Table 1, electron-withdrawing substituted phenylglyoxals such as 4-Cl and 4-Br worked as well as electron-donating substituted phenylglyoxals such as 3$\mathrm{MeO}, 4-\mathrm{MeO}$ and 3,4-(MeO $)_{2}$ in DABCO-catalyzed aldol reaction in water. Phenyl-, 4-Clphenyl- and 4-Br-phenylglyoxals underwent aldol reaction with excellent diastereoselectivity (3a-c and 3g,h), while electron-donating substituted phenylglyoxals afforded corresponding 2-hydroxy-1,4diketones $\mathbf{3 d}-\mathbf{f}$ and $\mathbf{3 i}$ as a mixture of two stereoisomers. As shown in Fig. 2, the syn/anti ratio was determined by ${ }^{1} \mathrm{H}$ NMR, using the intensity of the $H_{a}$ for two isomers. The coupling constant $\left({ }^{3} J_{\mathrm{Ha}, \mathrm{Hb}}\right)$ for signal of the anti-isomer is higher than that of the $s y n$-isomer. According to the ${ }^{1} \mathrm{H}$ NMR spectrum, the $H_{a}$ signal for the $s y n$-isomer has a higher $\delta$ value than that for the anti-isomer. For instance, in the ${ }^{1} \mathrm{H}$ NMR spectra of 2-hydroxy-1,3-bis(4-methoxyphenyl)pentane-1,4-dione (3d), the signal at $\delta=5.78 \mathrm{ppm}$ $\left({ }^{3} J_{\mathrm{Ha}, \mathrm{Hb}}=4.4 \mathrm{~Hz}\right)$ is contributed by the $s y n$-isomer, while the one at $5.28 \mathrm{ppm}\left({ }^{3} \mathrm{~J}_{\mathrm{Ha}, \mathrm{Hb}}=5.6 \mathrm{~Hz}\right)$ is contributed by the anti-isomer. 


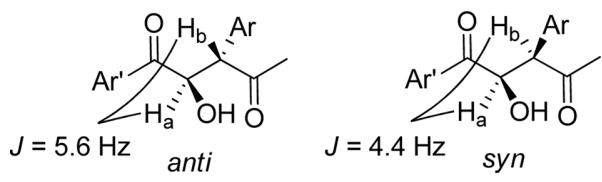

$3 d: A r=A r^{\prime}=4-\mathrm{MeOC}_{6} \mathrm{H}_{4}$

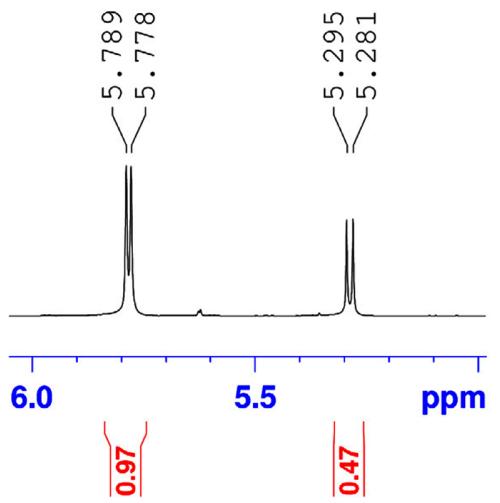

Fig. 2. Identification of anti and syn isomer by ${ }^{1} \mathrm{H}$ NMR.

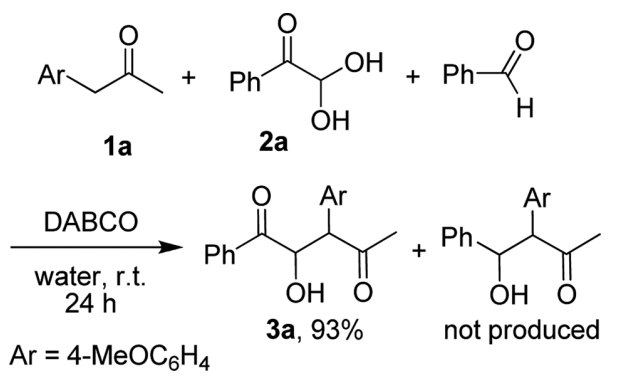

Scheme 3. Chemoselectivity of the aldol reaction of 1 a toward phenylglyoxal, in preference to benzaldehyde.

Another characteristic feature of the present protocol is the high chemoselectivity of the aldol reaction toward phenylglyoxal, in preference to benzaldehyde as shown in Scheme 3. When aldol reaction of phenylglyoxal with 1 a in the presence of 1 equiv. of benzaldehyde was carried out under same conditions, only $\mathbf{3 a}$ was isolated and benzaldehyde was recovered without changes.

The results encouraged us to work on the aldol reaction of acetophenone derivatives $\mathbf{4}$ with arylglyoxal hydrates 2 (Scheme 4). The reactions were conducted by stirring of a mixture of acetophenones and arylglyoxal hydrates in water at room temperature in the presence of catalytic

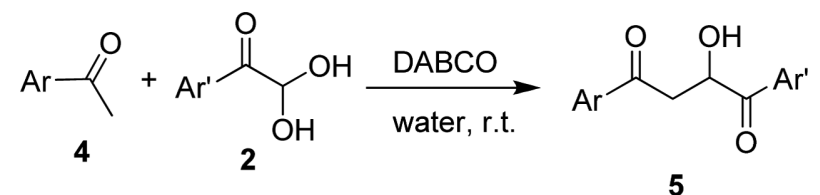

Scheme 4. DABCO-catalyzed aldol reaction of acetophenones 4 with $\mathbf{2}$ in water at room temperature.
Table 2. Aldol reaction of acetophenones 4 with arylglyoxal hydrates $\mathbf{2}$ in the presence of DABCO in water at room temperature (See also Scheme 4)

\begin{tabular}{ccccc}
\hline $\mathrm{Ar}^{\prime}[2]$ & $\mathrm{Ar}[4]$ & Product (5) & Time (h) & Yield (\%) $^{a}$ \\
\hline $\mathbf{2 a}$ & $4-\mathrm{ClC}_{6} \mathrm{H}_{4}$ & $\mathbf{5 a}$ & 28 & 59 \\
$\mathbf{2 a}$ & $4-\mathrm{BrC}_{6} \mathrm{H}_{4}$ & $\mathbf{5 b}$ & 15 & 25 \\
$\mathbf{2 a}$ & $4-\mathrm{NO}_{2} \mathrm{C}_{6} \mathrm{H}_{4}$ & $\mathbf{5 c}$ & 24 & 47 \\
$\mathbf{2 b}$ & $\mathrm{Ph}$ & $\mathbf{5 d}$ & 30 & 67 \\
$\mathbf{2 b}$ & $4-\mathrm{ClC}_{6} \mathrm{H}_{4}$ & $\mathbf{5 e}$ & 48 & 56 \\
$\mathbf{2 b}$ & $4-\mathrm{BrC}_{6} \mathrm{H}_{4}$ & $\mathbf{5 f}$ & 58 & 53 \\
$\mathbf{2 b}$ & $2-$ thienyl & $\mathbf{5 g}$ & 24 & 64 \\
$\mathbf{2 c}$ & $\mathrm{Ph}_{\mathbf{2 c}}$ & $\mathbf{5 h}$ & 48 & 59 \\
$\mathbf{2 c}$ & $4-\mathrm{ClC}_{6} \mathrm{H}_{4}$ & $\mathbf{5 i}$ & 48 & 60 \\
$\mathbf{2 c}$ & $4-\mathrm{BrC}_{6} \mathrm{H}_{4}$ & $\mathbf{5 j}$ & 192 & 55 \\
$\mathbf{2 c}$ & $2-\mathrm{NO}_{2} \mathrm{C}_{6} \mathrm{H}_{4}$ & $\mathbf{5 k}$ & 18 & 88 \\
\hline
\end{tabular}

${ }^{\bar{a}}$ Yields refer to isolated products by simple filtration of the reaction mixture and washing with warm water and cold $\mathrm{EtOH}$ to remove unreacted arylglyoxals and acetophenones, respectively.

amount of DABCO. The reaction mixture was solidified after appropriate time. The obtained solid was filtered off and washed with hot water to remove arylglyoxal and acetophenone residue. The results are summarized in Table 2.

As shown in Table 2, different 4 were subjected to DABCOcatalyzed aldol reaction with $\mathbf{2}$ to give corresponding 2hydroxy-1,4-diones 5 in 25-88\% yields. Also, heterocyclic methyl ketones such as 2-furyl and 2-thienyl methyl ketones underwent aldol reaction in water at room temperature to afford corresponding 1,4-diones 5g,l in good yields. The structure of products were established using FT-IR, ${ }^{1} \mathrm{H}$ NMR and ${ }^{13} \mathrm{C}$ NMR.

Attempts to aldol reaction of arylglyoxals with aliphatic ketones such as acetone and cyclohexanone under the same conditions were failed and a mixture of products was obtained.

\section{CONCLUSION}

In conclusion, a new green and simple methodology for construction of 1,4-diketone along with 2- and 3-hydroxy structural motifs was developed. Reactions were carried out in water at room temperature using DABCO as catalyst. Ketones such as $\mathbf{1 a , b}$ worked well in aldol reaction in water and afforded corresponding 2-hydroxy-1,4-diones in good to high yields. Electron-withdrawing substituted phenylgyloxals afforded the 1,4-diones with excellent syn selectivity, while arylglyoxals with electron-donating substituents gave corresponding 1,4-diones as a two diastereoisomers with ratio of about $2 / 1$ of syn/anti. Also, acetophenone 
deivatives worked well in aldol reaction with arylglyoxals in water.

\section{EXPERIMENTAL}

All chemicals were purchased and used without any further purification. NMR spectra were recorded at $400 \mathrm{MHz}$ for proton and at $100 \mathrm{MHz}$ for carbon nuclei in $\mathrm{CDCl}_{3}$ or DMSO- $d_{6}$. Most of compounds were new and characterized by their spectroscopic data (FT-IR, NMR and elemental analysis).

\section{General Procedure of the Aldol Reaction}

To a mixture of arylglyoxal $2(0.55 \mathrm{mmol})$ and ketone $\mathbf{1}$ or $4(0.5 \mathrm{mmol})$ in $3-5 \mathrm{~mL}$ water, was added $0.02 \mathrm{~g}$ DABCO and stirred at room temperature. The progress of the reaction was monitored by TLC ( $n$-hexane/ethyl acetate; $7 / 3$ ). After completion of the reaction, the products were isolated as solid from reaction mixture, which was filtered off and washed with hot water and cold $\mathrm{EtOH}$ to remove unreacted arylglyoxals and ketones, respectively. All obtained products are almost pure, but further purification for elemental analysis were carried out by recrystallization from EtOH.

\section{2-Hydroxy-3-(4-methoxyphenyl)-1-phenylpentane-1,4- dione (3a)}

White solid, m.p. $95-95.3{ }^{\circ} \mathrm{C}$. FT-IR (KBr) $v=3316$ $(\mathrm{O}-\mathrm{H})$, 3068, $2891(\mathrm{C}-\mathrm{H}), 1712,1674(\mathrm{C}=\mathrm{O}), 1645$ $(\mathrm{C}=\mathrm{C}), 1245(\mathrm{C}-\mathrm{O}) \mathrm{cm}^{-1} .{ }^{1} \mathrm{H}$ NMR $\left(500 \mathrm{MHz}, \mathrm{CDCl}_{3}\right): \delta$ $=7.90\left(\mathrm{dd},{ }^{3} J_{\mathrm{H}, \mathrm{H}}=7.9 \mathrm{~Hz},{ }^{4} J_{\mathrm{H}, \mathrm{H}}=0.8 \mathrm{~Hz}, 2 \mathrm{H}, \mathrm{CH}_{\mathrm{Ar}}\right), 7.66$ $\left(\mathrm{m}, 1 \mathrm{H}, \mathrm{CH}_{\mathrm{Ar}}\right), 7.53\left(\mathrm{t},{ }^{3} J_{\mathrm{H}, \mathrm{H}}=7.9 \mathrm{~Hz}, 2 \mathrm{H}, \mathrm{CH}_{\mathrm{Ar}}\right), 7.01(\mathrm{~d}$, $\left.{ }^{3} J_{\mathrm{H}, \mathrm{H}}=9.5 \mathrm{~Hz}, 2 \mathrm{H}, \mathrm{CH}_{\mathrm{Ar}}\right), 6.86\left(\mathrm{~d},{ }^{3} J_{\mathrm{H}, \mathrm{H}}=9.6 \mathrm{~Hz}, 2 \mathrm{H}\right.$, $\left.\mathrm{CH}_{\mathrm{Ar}}\right), 5.85\left(\mathrm{~d},{ }^{3} J_{\mathrm{H}, \mathrm{H}}=4.0 \mathrm{~Hz}, 1 \mathrm{H}, \mathrm{CH}-\mathrm{O}\right), 4.03\left(\mathrm{~d},{ }^{3} J_{\mathrm{H}, \mathrm{H}}=\right.$ $4.1 \mathrm{~Hz}, 1 \mathrm{H}, \mathrm{CH}$ ), 3.82 (s, $3 \mathrm{H}, \mathrm{OCH}_{3}$ ), 3.26 (s, br., $1 \mathrm{H}, \mathrm{OH}$, Exchange with $\left.\mathrm{D}_{2} \mathrm{O}\right), 2.14\left(\mathrm{~s}, 3 \mathrm{H}, \mathrm{CH}_{3}\right) \mathrm{ppm} .{ }^{13} \mathrm{C}$ NMR $\left(125 \mathrm{MHz}, \mathrm{CDCl}_{3}\right): \delta=206.8(\mathrm{C}=\mathrm{O}), 200.4(\mathrm{C}=\mathrm{O}), 159.9$, 134.8, 134.2, 131.5, 129.3, 129.0, 125.4, 114.6, 73.3, 62.1, 55.6, 29.5 ppm. Anal. Calcd for $\mathrm{C}_{18} \mathrm{H}_{18} \mathrm{O}_{4}$ (298.33): C 72.47, H 6.08; Found: C 72.51, H 6.10\%.

1-(4-Chlorophenyl)-2-hydroxy-3-(4-methoxyphenyl) pentane-1,4-dione (3b)

White solid, m.p. $119-123{ }^{\circ} \mathrm{C}$. FT-IR $(\mathrm{KBr}) \quad v=3355$ (br., O-H), 3071, $2943(\mathrm{C}-\mathrm{H}), 1716,1685(\mathrm{C}=\mathrm{O}), 1583$ $(\mathrm{C}=\mathrm{C}), 1244(\mathrm{C}-\mathrm{O}), 601(\mathrm{C}-\mathrm{Cl}) \mathrm{cm}^{-1} \cdot{ }^{1} \mathrm{H}$ NMR $(400$ $\left.\mathrm{MHz}, \mathrm{CDCl}_{3}\right) \delta=7.82\left(\mathrm{~d},{ }^{3} J_{\mathrm{H}, \mathrm{H}}=8.8 \mathrm{~Hz}, 2 \mathrm{H}, \mathrm{CH}_{\mathrm{Ar}}\right), 7.37$ $\left(\mathrm{d},{ }^{3} J_{\mathrm{H}, \mathrm{H}}=8.4 \mathrm{~Hz}, 2 \mathrm{H}, \mathrm{CH}_{\mathrm{Ar}}\right), 7.20\left(\mathrm{~d},{ }^{3} J_{\mathrm{H}, \mathrm{H}}=8.8 \mathrm{~Hz}, 2 \mathrm{H}\right.$, $\left.\mathrm{CH}_{\mathrm{Ar}}\right), 6.84\left(\mathrm{~d},{ }^{3} J_{\mathrm{H}, \mathrm{H}}=8.8 \mathrm{~Hz}, 2 \mathrm{H}, \mathrm{CH}_{\mathrm{Ar}}\right), 5.23\left(\mathrm{~d},{ }^{3} J_{\mathrm{H}, \mathrm{H}}=\right.$ $5.2 \mathrm{~Hz}, 1 \mathrm{H}, \mathrm{CH}-\mathrm{O}), 4.20\left(\mathrm{~d},{ }^{3} J_{\mathrm{H}, \mathrm{H}}=5.2 \mathrm{~Hz}, 1 \mathrm{H}, \mathrm{CH}\right), 3.80$ (s, $\left.3 \mathrm{H}, \mathrm{OCH}_{3}\right), 2.20\left(\mathrm{~s}, 3 \mathrm{H}, \mathrm{CH}_{3}\right) \mathrm{ppm} .{ }^{13} \mathrm{C}$ NMR $(100$
$\left.\mathrm{MHz}, \mathrm{CDCl}_{3}\right) \delta=209.6,199.0(\mathrm{C}=\mathrm{O}), 159.5,139.9,133.4$, 130.5, 130.3, 128.7, 126.1, 114.5, 76.1, 59.8, 55.3, 30.2 ppm. Anal. Calcd for $\mathrm{C}_{18} \mathrm{H}_{17} \mathrm{ClO}_{4}$ (332.78): $\mathrm{C} 64.97, \mathrm{H}$ 5.15; Found: C 64.90, H 5.30\%.

1-(4-Bromophenyl)-2-hydroxy-3-(4-methoxyphenyl) pentane-1,4-dione (3c)

White solid, m.p. $118.5-120.1^{\circ} \mathrm{C}$. FT-IR (KBr) $v=3335$ (br., O-H), 3069, $2941(\mathrm{C}-\mathrm{H}), 1695(\mathrm{C}=\mathrm{O}), 1578(\mathrm{C}=\mathrm{C})$, $1244(\mathrm{C}-\mathrm{O}), 579(\mathrm{C}-\mathrm{Br}) \mathrm{cm}^{-1}{ }^{1}{ }^{1} \mathrm{HNMR}\left(400 \mathrm{MHz}, \mathrm{CDCl}_{3}\right) \delta$ $=7.72-7.75\left(\mathrm{~m}, 2 \mathrm{H}, \mathrm{CH}_{\mathrm{Ar}}\right), 7.53-7.56\left(\mathrm{~m}, 2 \mathrm{H}, \mathrm{CH}_{\mathrm{Ar}}\right), 7.19$ $7.22\left(\mathrm{~m}, 2 \mathrm{H}, \mathrm{CH}_{\mathrm{Ar}}\right), 6.83-6.86\left(\mathrm{~m}, 2 \mathrm{H}, \mathrm{CH}_{\mathrm{Ar}}\right), 5.22-5.28$ (m, 1H, CH-O) 4.33 (m, br., $1 \mathrm{H}, \mathrm{OH}), 4.20\left(\mathrm{~d},{ }^{3} \mathrm{~J}_{\mathrm{H}, \mathrm{H}}=5.6\right.$ $\mathrm{Hz}, 1 \mathrm{H}, \mathrm{CH}), 3.80\left(\mathrm{~s}, 3 \mathrm{H}, \mathrm{OCH}_{3}\right), 2.20\left(\mathrm{~s}, 3 \mathrm{H}, \mathrm{CH}_{3}\right) \mathrm{ppm}$. ${ }^{13} \mathrm{C} \mathrm{NMR}\left(100 \mathrm{MHz}, \mathrm{CDCl}_{3}\right) \delta=209.6,199.3(\mathrm{C}=\mathrm{O}), 159.5$, 133.8, 131.7, 130.6, 130.3, 128.6, 126.1, 114.5, 76.1, 59.8, 55.3, $30.1 \mathrm{ppm}$. Anal. Calcd for $\mathrm{C}_{18} \mathrm{H}_{17} \mathrm{BrO}_{4}$ (377.23): C 57.31, H 4.54; Found: C 56.76, H 4.27\%.

2-Hydroxy-1,3-bis(4-methoxyphenyl)pentane-1,4dione (3d)

Syn/anti $=67 / 33$. Yellow oil, FT-IR $(\mathrm{KBr}) v=3442$ (br., O-H), 3040, $2943(\mathrm{C}-\mathrm{H}), 1706,1670(\mathrm{C}=\mathrm{O}), 1602(\mathrm{C}=\mathrm{C})$, $1255(\mathrm{C}-\mathrm{O}) \mathrm{cm}^{-1} .1 \mathrm{H} \mathrm{NMR}\left(400 \mathrm{MHz}, \mathrm{CDCl}_{3}\right) \delta=7.82-$ $7.92\left(2 \times \mathrm{m}, 2 \mathrm{H}, \mathrm{CH}_{\mathrm{Ar}}\right), 6.79-7.21\left(3 \times \mathrm{m}, 6 \mathrm{H}, \mathrm{CH}_{\mathrm{Ar}}\right), 5.78$ $\left(\mathrm{d},{ }^{3} J_{\mathrm{H}, \mathrm{H}}=4.4 \mathrm{~Hz}, 0.67 \mathrm{H}, \mathrm{CH}-\mathrm{O}\right.$, anti $), 5.28\left(\mathrm{~d},{ }^{3} J_{\mathrm{H}, \mathrm{H}}=5.6\right.$ $\mathrm{Hz}, 0.33 \mathrm{H}, \mathrm{CH}-\mathrm{O}, s y n), 4.16\left(\mathrm{~d},{ }^{3} \mathrm{~J}_{\mathrm{H}, \mathrm{H}}=5.6 \mathrm{~Hz}, 0.33 \mathrm{H}\right.$, $\mathrm{CH}$, syn $), 4.02\left(\mathrm{~d},{ }^{3} J_{\mathrm{H}, \mathrm{H}}=4.4 \mathrm{~Hz}, 0.67 \mathrm{H}, \mathrm{CH}\right.$, anti $), 3.84-$ 3.90 [2×S: $(3.90, \sim 2 \mathrm{H}$, anti; $3.84, \sim 1 \mathrm{H}$, syn $\left.), 3 \mathrm{H}, \mathrm{OCH}_{3}\right]$, 3.76-3.79 [2×S: $(3.79, \sim 2 \mathrm{H}$, anti; 3.76, $1 \mathrm{H}$, syn $), 3 \mathrm{H}$, $\left.\mathrm{OCH}_{3}\right], 2.12-2.17$ [2×S: $(2.17, \sim 1 \mathrm{H}$, syn $; 2.12, \sim 2 \mathrm{H}$, anti $)$, $\left.3 \mathrm{H}, \mathrm{CH}_{3}\right] \mathrm{ppm} .{ }^{13} \mathrm{C} \mathrm{NMR}\left(100 \mathrm{MHz}, \mathrm{CDCl}_{3}\right) \delta=209.3$, 206.6, 198.4, $198.2(\mathrm{C}=\mathrm{O}), 164.1,163.8,159.4,159.3$, $132.2,131.4,131.1,130.3,127.8,127.0,126.5,125.2$, 114.4, 114.17, 114.16, 113.7, 75.5, 72.5, 61.9, 60.3, 55.6, 55.5, 55.3, 55.2, 30.4, 29.2 ppm. Anal. Calcd for $\mathrm{C}_{19} \mathrm{H}_{20} \mathrm{O}_{5}$ (328.36): C 69.50, H 6.14; Found: C 69.83, H 6.21\%.

2-Hydroxy-1-(3-methoxyphenyl)-3-(4-methoxyphenyl)pentane-1,4-dione (3e)

Syn/anti $=67 / 33$. Yellow oil, FT-IR $(\mathrm{KBr}) v=3504$ (br., O-H), 3098, 2947 (C-H), 1693 (br., C=O), $1596(\mathrm{C}=\mathrm{C})$, $1253(\mathrm{C}-\mathrm{O}) \mathrm{cm}^{-1} .{ }^{1} \mathrm{H}$ NMR $\left(400 \mathrm{MHz}, \mathrm{CDCl}_{3}\right) \delta=6.78-$ $7.50\left(9 \times \mathrm{m}, 8 \mathrm{H}, \mathrm{CH}_{\mathrm{Ar}}\right), 5.82\left(\mathrm{~d},{ }^{3} J_{\mathrm{H}, \mathrm{H}}=4.0 \mathrm{~Hz}, 0.67 \mathrm{H}\right.$, $\mathrm{CH}-\mathrm{O}$, anti), $5.30\left(\mathrm{~d},{ }^{3} J_{\mathrm{H}, \mathrm{H}}=5.6 \mathrm{~Hz}, 0.33 \mathrm{H}, \mathrm{CH}-\mathrm{O}\right.$, syn), $4.19\left(\mathrm{~d},{ }^{3} J_{\mathrm{H}, \mathrm{H}}=5.6 \mathrm{~Hz}, 0.33 \mathrm{H}, \mathrm{CH}, \operatorname{syn}\right), 4.04\left(\mathrm{~d},{ }^{3} J_{\mathrm{H}, \mathrm{H}}=\right.$ $4.0 \mathrm{~Hz}, 0.67 \mathrm{H}, \mathrm{CH}$, anti), $3.83-3.87[2 \times \mathrm{S}:(3.87, \sim 2 \mathrm{H}$, anti; 3.83, $\sim 1 \mathrm{H}$, syn), $\left.3 \mathrm{H}, \mathrm{OCH}_{3}\right], 3.78-3.81$ [2× $\mathrm{S}:(3.81$, $\sim 2 \mathrm{H}$, anti; $3.78, \sim 1 \mathrm{H}$, syn), $\left.3 \mathrm{H}, \mathrm{OCH}_{3}\right], 2.13-2.17$ [2×S: (2.17, $\sim 1 \mathrm{H}$, syn; $2.13, \sim 2 \mathrm{H}$, anti), $\left.3 \mathrm{H}, \mathrm{CH}_{3}\right] \mathrm{ppm} .{ }^{13} \mathrm{C} \mathrm{NMR}$ $\left(100 \mathrm{MHz}, \mathrm{CDCl}_{3}\right) \delta=209.3,206.4,200.0,199.8(\mathrm{C}=\mathrm{O})$, $160.0,159.6,159.5,159.4,136.3,135.6,131.1,130.3$, 
$130.0,129.4,126.4,125.0,121.6,121.1,120.3,120.2$, 114.4, 114.2, 113.0, 112.9, 75.9, 72.9, 61.7, 60.3, 55.5, 55.4, 55.3, 55.2, 30.2, $29.1 \mathrm{ppm}$. Anal. Calcd for $\mathrm{C}_{19} \mathrm{H}_{20} \mathrm{O}_{5}$ (328.36): C 69.50, H 6.14; Found: C 68.89, H 5.78\%.

1-(3,4-Dimetoxyphenyl)-2-hydroxy-3-(4-metoxyphenyl)pentane-1,4-dione (3f)

Syn/anti $=64 / 36$. Yellow oil, FT-IR $(\mathrm{KBr}) v=3511$ (br., $\mathrm{O}-\mathrm{H}), 3093,2941(\mathrm{C}-\mathrm{H}), 1712,1672(\mathrm{C}=\mathrm{O}), 1598(\mathrm{C}=\mathrm{C})$, $1265(\mathrm{C}-\mathrm{O}) \mathrm{cm}^{-1} .{ }^{1} \mathrm{H}$ NMR $\left(400 \mathrm{MHz}, \mathrm{CDCl}_{3}\right) \delta=7.51-$ $7.61\left[2 \times \mathrm{dd}: 7.59\left(\mathrm{dd},{ }^{3} \mathrm{~J}_{\mathrm{H}, \mathrm{H}}=8.4 \mathrm{~Hz},{ }^{4} J_{\mathrm{H}, \mathrm{H}}=2.0 \mathrm{~Hz}\right), 7.50\right.$ $\left.\left(\mathrm{dd},{ }^{3} J_{\mathrm{H}, \mathrm{H}}=8.4 \mathrm{~Hz},{ }^{4} J_{\mathrm{H}, \mathrm{H}}=2.0 \mathrm{~Hz}\right) 1 \mathrm{H}, \mathrm{CH}_{\mathrm{Ar}}\right], 7.39-7.41$ $\left(\mathrm{m}, 1 \mathrm{H}, \mathrm{CH}_{\mathrm{Ar}}\right), 7.12-7.20\left[2 \times \mathrm{d}: 7.20\left(\mathrm{~d},{ }^{3} J_{\mathrm{H}, \mathrm{H}}=8.8 \mathrm{~Hz}\right)\right.$, $\left.7.12\left(\mathrm{~d},{ }^{3} J_{\mathrm{H}, \mathrm{H}}=8.8 \mathrm{~Hz}\right), 1 \mathrm{H}, \mathrm{CH}_{\mathrm{Ar}}\right], 6.93-7.02[2 \times \mathrm{d}: 7.02$ $\left.\left(\mathrm{d},{ }^{3} J_{\mathrm{H}, \mathrm{H}}=8.8 \mathrm{~Hz}\right), 6.93\left(\mathrm{~d},{ }^{3} J_{\mathrm{H}, \mathrm{H}}=8.4 \mathrm{~Hz}\right), 2 \mathrm{H}, \mathrm{CH}_{\mathrm{Ar}}\right]$, 6.79-6.85 (m, 2H, $\left.\mathrm{CH}_{\mathrm{Ar}}\right), 5.78\left(\mathrm{~d},{ }^{3} J_{\mathrm{H}, \mathrm{H}}=4.4 \mathrm{~Hz}, 0.64 \mathrm{H}\right.$, $\mathrm{CH}-\mathrm{O}$, anti), $5.29\left(\mathrm{~d},{ }^{3} J_{\mathrm{H}, \mathrm{H}}=5.6 \mathrm{~Hz}, 0.36 \mathrm{H}, \mathrm{CH}-\mathrm{O}\right.$, syn $)$, $4.18\left(\mathrm{~d},{ }^{3} J_{\mathrm{H}, \mathrm{H}}=5.6 \mathrm{~Hz}, 0.36 \mathrm{H}, \mathrm{CH}, s y n\right), 4.04\left(\mathrm{~d},{ }^{3} J_{\mathrm{H}, \mathrm{H}}=\right.$ $4.4 \mathrm{~Hz}, 0.64 \mathrm{H}, \mathrm{CH}$, anti), $3.88-3.97\left(3 \times \mathrm{s}, 6 \mathrm{H}, \mathrm{OCH}_{3}\right)$, $3.73-3.79[2 \times \mathrm{S}:(3.79, \sim 1.9 \mathrm{H}$, anti; $3.73, \sim 1.1 \mathrm{H}$, syn $), 3 \mathrm{H}$, $\left.\mathrm{OCH}_{3}\right], 2.12-2.17$ [2×S: $(2.17, \sim 1.1 \mathrm{H}, \operatorname{syn} ; 2.12, \sim 1.9 \mathrm{H}$, anti), $3 \mathrm{H}, \mathrm{CH}_{3}$ ] ppm. ${ }^{13} \mathrm{C} \mathrm{NMR}\left(100 \mathrm{MHz}, \mathrm{CDCl}_{3}\right) \delta=$ 209.5, 206.7, 198.4, $198.2(\mathrm{C}=\mathrm{O}), 159.4,159.36,153.9$, 153.6, 149.3, 148.8, 131.1, 130.4, 127.9, 127.2, 126.4, 125.3, $124.1,123.4,114.4,114.2,110.9,110.7,110.3,109.9$, 75.5, 72.5, 62.1, 60.3, 56.2, 56.1, 55.9, 55.3, 55.2, 50.1, 30.3, 29.2 ppm. Anal. Calcd for $\mathrm{C}_{20} \mathrm{H}_{22} \mathrm{O}_{6}$ (358.39): C 67.03, H 6.19; Found: C 66.51, H 6.28\%.

1-(4-Chlorophenyl)-2-hydroxy-3,4-diphenylbutane1,4-dione (3g)

White solid, m.p. $176.8-179.6^{\circ} \mathrm{C}$. FT-IR $(\mathrm{KBr}) v=3367$ (br., $\mathrm{O}-\mathrm{H}), 3060,2893(\mathrm{C}-\mathrm{H}), 1670(\mathrm{C}=\mathrm{O}), 1583(\mathrm{C}=\mathrm{C})$, $1199(\mathrm{C}-\mathrm{O}), 694(\mathrm{C}-\mathrm{Cl}) \mathrm{cm}^{-1} .{ }^{1} \mathrm{H} \mathrm{NMR}\left(400 \mathrm{MHz}, \mathrm{CDCl}_{3}\right)$ $\delta=7.97\left(\mathrm{~d},{ }^{3} J_{\mathrm{H}, \mathrm{H}}=8.0 \mathrm{~Hz}, 2 \mathrm{H}, \mathrm{CH}_{\mathrm{Ar}}\right), 7.85\left(\mathrm{~d},{ }^{3} J_{\mathrm{H}, \mathrm{H}}=8.8\right.$ $\left.\mathrm{Hz}, 2 \mathrm{H}, \mathrm{CH}_{\mathrm{Ar}}\right), 7.52-7.56\left(\mathrm{~m}, 1 \mathrm{H}, \mathrm{CH}_{\mathrm{Ar}}\right), 7.41-7.44(\mathrm{~m}$, $\left.2 \mathrm{H}, \mathrm{CH}_{\mathrm{Ar}}\right), 7.19-7.36\left(\mathrm{~m}, 7 \mathrm{H}, \mathrm{CH}_{\mathrm{Ar}}\right), 5.44-5.48\left(\mathrm{dd},{ }^{3} \mathrm{~J}_{\mathrm{H}, \mathrm{H}}\right.$ $=5.6,9.2 \mathrm{~Hz}, 1 \mathrm{H}, \mathrm{CH}-\mathrm{O}), 5.13\left(\mathrm{~d},{ }^{3} J_{\mathrm{H}, \mathrm{H}}=5.6 \mathrm{~Hz}, 1 \mathrm{H}\right.$, $\mathrm{CH}), 4.53\left(\mathrm{~d},{ }^{3} J_{\mathrm{H}, \mathrm{H}}=9.2 \mathrm{~Hz}, 1 \mathrm{H}, \mathrm{OH}\right) \mathrm{ppm} .{ }^{13} \mathrm{C} \mathrm{NMR}(100$ $\left.\mathrm{MHz}, \mathrm{CDCl}_{3}\right) \delta=200.4,199.3(\mathrm{C}=\mathrm{O}), 139.9,136.2,134.5$, 133.6, 133.5, 130.7, 129.2, 129.0, 128.9, 128.7, 128.0, 59.5, 55.7, 31.3 ppm. Anal. Calcd for $\mathrm{C}_{22} \mathrm{H}_{17} \mathrm{ClO}_{3}$ (364.82): C 72.43, H 4.70; Found: C 72.50, H 4.80\%.

1-(4-Bromophenyl)-2-hydroxy-3,4-diphenylbutane-

\section{1,4-dione (3h)}

White solid, m.p. $180-184.5^{\circ} \mathrm{C}$. FT-IR $(\mathrm{KBr}) v=3361$ (br., O-H), 3059, $2914(\mathrm{C}-\mathrm{H}), 1666(\mathrm{C}=\mathrm{O}), 1577(\mathrm{C}=\mathrm{C})$, $1199(\mathrm{C}-\mathrm{O}), 702(\mathrm{C}-\mathrm{Br}) \mathrm{cm}^{-1} .{ }^{1} \mathrm{H} \mathrm{NMR}\left(400 \mathrm{MHz}, \mathrm{CDCl}_{3}\right)$ $\delta=7.97\left(\mathrm{~d},{ }^{3} J_{\mathrm{H}, \mathrm{H}}=7.6 \mathrm{~Hz}, 2 \mathrm{H}, \mathrm{CH}_{\mathrm{Ar}}\right), 7.77\left(\mathrm{~d},{ }^{3} J_{\mathrm{H}, \mathrm{H}}=7.6\right.$ $\left.\mathrm{Hz}, 2 \mathrm{H}, \mathrm{CH}_{\mathrm{Ar}}\right), 7.51-7.56\left(\mathrm{~m}, 3 \mathrm{H}, \mathrm{CH}_{\mathrm{Ar}}\right), 7.41-7.44$ (m, $\left.2 \mathrm{H}, \mathrm{CH}_{\mathrm{Ar}}\right), 7.19-7.33\left(\mathrm{~m}, 5 \mathrm{H}, \mathrm{CH}_{\mathrm{Ar}}\right), 5.45(\mathrm{~m}, 1 \mathrm{H}, \mathrm{CH}-\mathrm{O})$, $5.13\left(\mathrm{~d},{ }^{3} J_{\mathrm{H}, \mathrm{H}}=6.0 \mathrm{~Hz}, 1 \mathrm{H}, \mathrm{CH}\right), 4.53\left(\mathrm{~d},{ }^{3} J_{\mathrm{H}, \mathrm{H}}=7.6 \mathrm{~Hz}\right.$, $1 \mathrm{H}, \mathrm{OH}) \mathrm{ppm} .{ }^{1} \mathrm{H} \mathrm{NMR}\left(400 \mathrm{MHz}, \mathrm{CDCl}_{3}+\right.$ drops of $\mathrm{D}_{2} \mathrm{O}$ ) $\delta=7.97\left(\mathrm{~d},{ }^{3} J_{\mathrm{H}, \mathrm{H}}=8.8 \mathrm{~Hz}, 2 \mathrm{H}, \mathrm{CH}_{\mathrm{Ar}}\right), 7.77\left(\mathrm{~d},{ }^{3} J_{\mathrm{H}, \mathrm{H}}=8.8\right.$ $\left.\mathrm{Hz}, 2 \mathrm{H}, \mathrm{CH}_{\mathrm{Ar}}\right), 7.51-7.56\left(\mathrm{~m}, 3 \mathrm{H}, \mathrm{CH}_{\mathrm{Ar}}\right), 7.41-7.44(\mathrm{~m}$, $\left.2 \mathrm{H}, \mathrm{CH}_{\mathrm{Ar}}\right), 7.19-7.33\left(\mathrm{~m}, 5 \mathrm{H}, \mathrm{CH}_{\mathrm{Ar}}\right), 5.43\left(\mathrm{~d},{ }^{3} \mathrm{~J}_{\mathrm{H}, \mathrm{H}}=5.6\right.$ $\mathrm{Hz}, 1 \mathrm{H}, \mathrm{CHO}), 5.13\left(\mathrm{~d},{ }^{3} J_{\mathrm{H}, \mathrm{H}}=5.6 \mathrm{~Hz}, 1 \mathrm{H}, \mathrm{CH}\right) \mathrm{ppm} .{ }^{13} \mathrm{C}$ NMR $\left(100 \mathrm{MHz}, \mathrm{CDCl}_{3}\right) \delta=200.5,199.5(\mathrm{C}=\mathrm{O}), 136.2$, 134.5, 133.9, 133.6, 131.7, 130.7, 129.2, 129.0, 128.9, $128.7,128.6,128.0,58.4,55.6,31.0 \mathrm{ppm}$. Anal. Calcd for $\mathrm{C}_{22} \mathrm{H}_{17} \mathrm{BrO}_{3}$ (409.27): C 64.56, H 4.19; Found: C 64.01, H $4.72 \%$.

\section{2-Hydroxy-1-(4-methoxyphenyl)-3,4-diphenylbutane-}

\section{1,4-dione (3i)}

Syn/anti $=65 / 35$. White solid. FT-IR $(\mathrm{KBr}) v=3471(\mathrm{O}-$ $\mathrm{H})$, 3064, $2952(\mathrm{C}-\mathrm{H}), 1670(\mathrm{C}=\mathrm{O}), 1591(\mathrm{C}=\mathrm{C}), 1267$ $(\mathrm{C}-\mathrm{O}) \mathrm{cm}^{-1} .{ }^{1} \mathrm{H}$ NMR $\left(400 \mathrm{MHz}, \mathrm{CDCl}_{3}\right) \delta=7.96-7.99$ $\left(\mathrm{m}, 2 \mathrm{H}, \mathrm{CH}_{\mathrm{Ar}}\right), 7.86-7.90\left(\mathrm{~m}, 2 \mathrm{H}, \mathrm{CH}_{\mathrm{Ar}}\right), 7.16-7.55$ $\left(7 \times \mathrm{m}, 8 \mathrm{H}, \mathrm{CH}_{\mathrm{Ar}}\right), 6.83-6.98\left[2 \times \mathrm{d}:\left(6.98,{ }^{3} J_{\mathrm{H}, \mathrm{H}}=9.2 \mathrm{~Hz}\right.\right.$; $\left.\left.6.83,{ }^{3} \mathrm{~J}_{\mathrm{H}, \mathrm{H}}=8.8 \mathrm{~Hz}\right), 2 \mathrm{H}, \mathrm{CH}_{\mathrm{Ar}}\right), 5.84(\mathrm{~m}, \mathrm{br} ., 0.65 \mathrm{H}, \mathrm{CH}-$ $\mathrm{O}$, anti), $5.53(\mathrm{~m}$, br., $0.35 \mathrm{H}, \mathrm{CH}-\mathrm{O}$, syn $), 5.09\left(\mathrm{~d},{ }^{3} J_{\mathrm{H}, \mathrm{H}}=\right.$ $6.4 \mathrm{~Hz}, 0.35 \mathrm{H}, \mathrm{CH}, s y n), 5.05\left(\mathrm{~d},{ }^{3} J_{\mathrm{H}, \mathrm{H}}=5.2 \mathrm{~Hz}, 0.65 \mathrm{H}\right.$, $\mathrm{CH}$, anti), 4.45 (m, br., $0.35 \mathrm{H}, \mathrm{OH}$, syn), 3.86-3.92 [2×S: (3.92, 2 $\mathrm{H}$, anti; 3.86, 1H, syn), 3H, $\left.\mathrm{OCH}_{3}\right], 3.03$ (s, br., $0.65 \mathrm{H}, \mathrm{OH}$, anti) ppm. ${ }^{13} \mathrm{C} \mathrm{NMR}\left(100 \mathrm{MHz}, \mathrm{CDCl}_{3}\right) \delta=$ 200.0, 198.7, 198.0, $197.9(\mathrm{C}=\mathrm{O}), 164.1,163.8,136.5,136.0$, 134.1, 133.4, 133.1, 131.6, 131.4, 129.7, 129.1, 129.0, 128.97, 128.9, 128.6, 128.5, 127.9, 127.8, 127.5, 114.1, 113.6, 76.3, 73.6, 57.5, 56.2, 55.6, $55.5 \mathrm{ppm}$. Anal. Calcd for $\mathrm{C}_{23} \mathrm{H}_{20} \mathrm{O}_{4}$ (360.40): C 76.65, H 5.59; Found: C 76.09, H 5.72\%.

4-(4-Chlorophenyl)-2-hydroxy-1-phenylbutane-1,4dione (5a)

White solid, m.p. $132.5-136^{\circ} \mathrm{C}$. FT-IR $(\mathrm{KBr}) v=3743$ $(\mathrm{O}-\mathrm{H}), 3061(\mathrm{C}-\mathrm{H}), 1681(\mathrm{C}=\mathrm{O}), 1552(\mathrm{C}=\mathrm{C}), 1195(\mathrm{C}-\mathrm{O})$, $651(\mathrm{CCl}) \mathrm{cm}^{-1} .{ }^{1} \mathrm{H}$ NMR $\left(400 \mathrm{MHz}, \mathrm{CDCl}_{3}\right) \delta=8.01(\mathrm{~d}$, $\left.{ }^{3} J_{\mathrm{H}, \mathrm{H}}=8.4 \mathrm{~Hz}, 2 \mathrm{H}, \mathrm{CH}_{\mathrm{Ar}}\right), 7.91\left(\mathrm{~d},{ }^{3} J_{\mathrm{H}, \mathrm{H}}=8.4 \mathrm{~Hz}, 2 \mathrm{H}\right.$, $\left.\mathrm{CH}_{\mathrm{Ar}}\right), 7.66\left(\mathrm{t},{ }^{3} J_{\mathrm{H}, \mathrm{H}}=7.2 \mathrm{~Hz}, 1 \mathrm{H}, \mathrm{CH}_{\mathrm{Ar}}\right), 7.54\left(\mathrm{t},{ }^{3} \mathrm{~J}_{\mathrm{H}, \mathrm{H}}=\right.$ $\left.7.6 \mathrm{~Hz}, 2 \mathrm{H}, \mathrm{CH}_{\mathrm{Ar}}\right), 7.46\left(\mathrm{~d},{ }^{3} \mathrm{~J}_{\mathrm{H}, \mathrm{H}}=8.8 \mathrm{~Hz}, 2 \mathrm{H}, \mathrm{CH}_{\mathrm{Ar}}\right), 5.69$ (s, br., $1 \mathrm{H}, \mathrm{OH}), 4.05(\mathrm{~m}, 1 \mathrm{H}, \mathrm{CH}-\mathrm{O}), 3.33-3.45(\mathrm{~m}, 2 \mathrm{H}$, $\left.\mathrm{CH}_{2}\right) \mathrm{ppm} .{ }^{1} \mathrm{H}$ NMR $\left(400 \mathrm{MHz}, \mathrm{DMSO}-d_{6}\right) \delta=8.03(\mathrm{~d}$, $\left.{ }^{3} J_{\mathrm{H}, \mathrm{H}}=7.2 \mathrm{~Hz}, 2 \mathrm{H}, \mathrm{CH}_{\mathrm{Ar}}\right), 8.00\left(\mathrm{~d},{ }^{3} \mathrm{~J}_{\mathrm{H}, \mathrm{H}}=8.4 \mathrm{~Hz}, 2 \mathrm{H}\right.$, $\left.\mathrm{CH}_{\mathrm{Ar}}\right), 7.67\left(\mathrm{t},{ }^{3} \mathrm{~J}_{\mathrm{H}, \mathrm{H}}=7.2 \mathrm{~Hz}, 1 \mathrm{H}, \mathrm{CH}_{\mathrm{Ar}}\right), 7.62\left(\mathrm{~d},{ }^{3} \mathrm{~J}_{\mathrm{H}, \mathrm{H}}=\right.$ $\left.8.4 \mathrm{~Hz}, 2 \mathrm{H}, \mathrm{CH}_{\mathrm{Ar}}\right), 7.56\left(\mathrm{t},{ }^{3} \mathrm{~J}_{\mathrm{H}, \mathrm{H}}=7.6 \mathrm{~Hz}, 2 \mathrm{H}, \mathrm{CH}_{\mathrm{Ar}}\right), 5.85$ $\left(\mathrm{d},{ }^{3} J_{\mathrm{H}, \mathrm{H}}=7.6 \mathrm{~Hz}, 1 \mathrm{H}, \mathrm{OH}\right), 5.43-5.48(\mathrm{~m}, 1 \mathrm{H}, \mathrm{CH}-\mathrm{O})$, $3.35-3.59$ [2×dd: $3.54-3.59\left(\mathrm{dd},{ }^{3} J_{\mathrm{H}, \mathrm{H}}=6.8,17.2 \mathrm{~Hz}, 1 \mathrm{H}\right.$, $\left.\left.\mathrm{CH}_{2}\right), 3.35-3.41\left(\mathrm{dd},{ }^{3} \mathrm{~J}_{\mathrm{H}, \mathrm{H}}=6.4,17.2 \mathrm{~Hz}, 1 \mathrm{H}, \mathrm{CH}_{2}\right)\right] \mathrm{ppm}$. ${ }^{13} \mathrm{C}$ NMR (100 MHz, DMSO- $\left.d_{6}\right) \delta=199.5,197.5(\mathrm{C}=\mathrm{O})$, 138.8, 135.7, 135.5, 133.7, 130.5, 129.3, 129.2, 129.17, 69.1, $42.6 \mathrm{ppm}$. Anal. Calcd for $\mathrm{C}_{16} \mathrm{H}_{13} \mathrm{ClO}_{3}$ (288.73): $\mathrm{C} 66.56$, H 4.54; Found: C 66.49, H 4.51\%. 


\section{4-(4-Bromophenyl)-2-hydroxy-1-phenylbutane-1,4- dione $(5 b)$}

White solid, m.p. $145.8-149.6{ }^{\circ} \mathrm{C}$. FT-IR (KBr) $v=3448$ (br., O-H), 3058, $2964(\mathrm{C}-\mathrm{H}), 1676(\mathrm{C}=\mathrm{O}), 1583(\mathrm{C}=\mathrm{C})$, $1191(\mathrm{C}-\mathrm{O}), 696(\mathrm{C}-\mathrm{Br}) \mathrm{cm}^{-1} .{ }^{1} \mathrm{H}$ NMR $\left(400 \mathrm{MHz}, \mathrm{CDCl}_{3}\right)$ $\delta=8.01\left(\mathrm{~d},{ }^{3} J_{\mathrm{H}, \mathrm{H}}=7.6 \mathrm{~Hz}, 2 \mathrm{H}, \mathrm{CH}_{\mathrm{Ar}}\right), 7.83\left(\mathrm{~d},{ }^{3} J_{\mathrm{H}, \mathrm{H}}=8.4\right.$ $\left.\mathrm{Hz}, 2 \mathrm{H}, \mathrm{CH}_{\mathrm{Ar}}\right), 7.61-7.67\left(\mathrm{~m}, 3 \mathrm{H}, \mathrm{CH}_{\mathrm{Ar}}\right), 7.54\left(\mathrm{t},{ }^{3} \mathrm{~J}_{\mathrm{H}, \mathrm{H}}=\right.$ $\left.7.6 \mathrm{~Hz}, 2 \mathrm{H}, \mathrm{CH}_{\mathrm{Ar}}\right), 5.68(\mathrm{~m}, 1 \mathrm{H}, \mathrm{CH}-\mathrm{O}), 4.04\left(\mathrm{~d},{ }^{3} \mathrm{~J}_{\mathrm{H}, \mathrm{H}}=\right.$ $6.0 \mathrm{~Hz}, 1 \mathrm{H}, \mathrm{OH}), 3.33-3.44\left(\mathrm{~m}, 2 \mathrm{H}, \mathrm{CH}_{2}\right) \mathrm{ppm} .{ }^{13} \mathrm{C} \mathrm{NMR}$ $\left(100 \mathrm{MHz}, \mathrm{CDCl}_{3}\right) \delta=200.6,196.3(\mathrm{C}=\mathrm{O}), 135.4,134.1$, 133.5, 132.0, 129.9, 129.0, 128.9, 128.7, 70.1, 43.5 ppm. Anal. Calcd for $\mathrm{C}_{16} \mathrm{H}_{13} \mathrm{BrO}_{3}$ (333.18): C 57.68, H 3.93; Found: C 57.50, H 4.00\%.

2-Hydroxy-4-(4-nitrophenyl)-1-phenylbutane-1,4dione (5c)

Yellow solid, m.p. $128-132{ }^{\circ} \mathrm{C}$. FT-IR (KBr) $v=3450$ $(\mathrm{O}-\mathrm{H}), 3076(\mathrm{C}-\mathrm{H}), 1670(\mathrm{C}=\mathrm{O}), 1593(\mathrm{C}=\mathrm{C}), 1521$, $1315\left(\mathrm{NO}_{2}\right), 1195(\mathrm{C}-\mathrm{O}) \mathrm{cm}^{-1} .{ }^{1} \mathrm{H} \mathrm{NMR}\left(400 \mathrm{MHz}, \mathrm{CDCl}_{3}\right)$ $\delta=8.34\left(\mathrm{~d},{ }^{3} J_{\mathrm{H}, \mathrm{H}}=8.8 \mathrm{~Hz}, 2 \mathrm{H}, \mathrm{CH}_{\mathrm{Ar}}\right), 8.13\left(\mathrm{~d},{ }^{3} J_{\mathrm{H}, \mathrm{H}}=8.8\right.$ $\left.\mathrm{Hz}, 2 \mathrm{H}, \mathrm{CH}_{\mathrm{Ar}}\right), 8.02\left(\mathrm{~d},{ }^{3} \mathrm{~J}_{\mathrm{H}, \mathrm{H}}=7.6 \mathrm{~Hz}, 2 \mathrm{H}, \mathrm{CH}_{\mathrm{Ar}}\right), 7.67(\mathrm{t}$, $\left.{ }^{3} J_{\mathrm{H}, \mathrm{H}}=7.6 \mathrm{~Hz}, 1 \mathrm{H}, \mathrm{CH}_{\mathrm{Ar}}\right), 7.54-7.58\left(\mathrm{~m}, 2 \mathrm{H}, \mathrm{CH}_{\mathrm{Ar}}\right), 5.68-$ $5.72(\mathrm{~m}, 1 \mathrm{H}, \mathrm{CH}-\mathrm{O}), 4.05\left(\mathrm{~d},{ }^{3} J_{\mathrm{H}, \mathrm{H}}=6.0 \mathrm{~Hz}, 1 \mathrm{H}, \mathrm{OH}\right), 3.41-$ $3.47\left(\mathrm{~m}, 2 \mathrm{H}, \mathrm{CH}_{2}\right) \mathrm{ppm} .{ }^{13} \mathrm{C} \mathrm{NMR}\left(100 \mathrm{MHz}, \mathrm{CDCl}_{3}\right) \delta=$ 200.2, $195.9(\mathrm{C}=\mathrm{O}), 150.6,141.1,134.3,133.3,129.4,129.1$, 128.7, 123.9, 70.0, 44.1 ppm. Anal. Calcd for $\mathrm{C}_{16} \mathrm{H}_{13} \mathrm{NO}_{5}$ (299.28): C 64.21, H 4.38, N 4.68; Found: C 64.18, H 4.31, N 4.55\%.

1-(4-Chlorophenyl)-2-hydroxy-4-phenylbutane-1,4dione (5d)

White solid, m.p. $151-155^{\circ} \mathrm{C}$. FT-IR (KBr) $v=3440(\mathrm{O}-$ $\mathrm{H}), 3080(\mathrm{C}-\mathrm{H}), 1679(\mathrm{C}=\mathrm{O}), 1554(\mathrm{C}=\mathrm{C}), 1190(\mathrm{C}-\mathrm{O})$, $750(\mathrm{C}-\mathrm{Cl}) \mathrm{cm}^{-1} .{ }^{1} \mathrm{H}$ NMR $\left(400 \mathrm{MHz}, \mathrm{CDCl}_{3}\right) \delta=7.96-$ $8.00\left(\mathrm{~m}, 4 \mathrm{H}, \mathrm{CH}_{\mathrm{Ar}}\right), 7.62\left(\mathrm{t},{ }^{3} J_{\mathrm{H}, \mathrm{H}}=7.6 \mathrm{~Hz}, 1 \mathrm{H}, \mathrm{CH}_{\mathrm{Ar}}\right)$, 7.48-7.52 (m, 4H, $\mathrm{CH}_{\mathrm{Ar}}$ ), 5.59-5.63 (m, 1H, CH-O), 4.02 $\left(\mathrm{d},{ }^{3} J_{\mathrm{H}, \mathrm{H}}=6.4 \mathrm{~Hz}, 1 \mathrm{H}, \mathrm{OH}\right), 3.38-3.52[2 \times \mathrm{dd}: 3.46-3.52$ $\left(\mathrm{dd},{ }^{3} J_{\mathrm{H}, \mathrm{H}}=6.8,17.2 \mathrm{~Hz}, 1 \mathrm{H}, \mathrm{CH}_{2}\right), 3.38-3.44\left(\mathrm{dd},{ }^{3} J_{\mathrm{H}, \mathrm{H}}=\right.$ 3.6, $\left.\left.17.2 \mathrm{~Hz}, 1 \mathrm{H}, \mathrm{CH}_{2}\right)\right]$ ppm. ${ }^{13} \mathrm{C} \mathrm{NMR}\left(100 \mathrm{MHz}, \mathrm{CDCl}_{3}\right)$ $\delta=199.5,197.5(\mathrm{C}=\mathrm{O}), 140.4,136.5,133.8,132.1,130.3$, 129.3, 128.8, 128.3, 70.4, $43.2 \mathrm{ppm}$. Anal. Calcd for $\mathrm{C}_{16} \mathrm{H}_{13} \mathrm{ClO}_{3}$ (288.73): C 66.56, H 4.54; Found: C 66.50, H $4.50 \%$.

1,4-Bis(4-chlorophenyl)-2-hydroxybutane-1,4-dione (5e) White solid, m.p. $159-162{ }^{\circ} \mathrm{C}$. FT-IR (KBr) $v=3427$ $(\mathrm{O}-\mathrm{H}), 3071(\mathrm{C}-\mathrm{H}), 1673(\mathrm{C}=\mathrm{O}), 1588(\mathrm{C}=\mathrm{C}), 1200(\mathrm{C}-$ O), $745(\mathrm{C}-\mathrm{Cl}) \mathrm{cm}^{-1}$. ${ }^{1} \mathrm{H}$ NMR $\left(400 \mathrm{MHz}, \mathrm{CDCl}_{3}\right) \delta=$ $7.98\left(\mathrm{~d},{ }^{3} J_{\mathrm{H}, \mathrm{H}}=8.8 \mathrm{~Hz}, 2 \mathrm{H}, \mathrm{CH}_{\mathrm{Ar}}\right), 7.91\left(\mathrm{~d},{ }^{3} J_{\mathrm{H}, \mathrm{H}}=8.8 \mathrm{~Hz}\right.$, $\left.2 \mathrm{H}, \mathrm{CH}_{\mathrm{Ar}}\right), 7.51\left(\mathrm{~d},{ }^{3} J_{\mathrm{H}, \mathrm{H}}=8.8 \mathrm{~Hz}, 2 \mathrm{H}, \mathrm{CH}_{\mathrm{Ar}}\right), 7.47(\mathrm{~d}$, $\left.{ }^{3} J_{\mathrm{H}, \mathrm{H}}=8.4 \mathrm{~Hz}, 2 \mathrm{H}, \mathrm{CH}_{\mathrm{Ar}}\right), 5.57-5.61(\mathrm{~m}, 1 \mathrm{H}, \mathrm{CH}-\mathrm{O})$, $4.01\left(\mathrm{~d},{ }^{3} J_{\mathrm{H}, \mathrm{H}}=6.4 \mathrm{~Hz}, 1 \mathrm{H}, \mathrm{OH}\right), 3.34-3.47$ [2×dd: $3.41-$ $3.47\left(\mathrm{dd},{ }^{3} J_{\mathrm{H}, \mathrm{H}}=7.2,17.2 \mathrm{~Hz}, 1 \mathrm{H}, \mathrm{CH}_{2}\right), 3.34-3.39(\mathrm{dd}$, $\left.\left.{ }^{3} J_{\mathrm{H}, \mathrm{H}}=3.6,17.2 \mathrm{~Hz}, 1 \mathrm{H}, \mathrm{CH}_{2}\right)\right] \mathrm{ppm} .{ }^{13} \mathrm{C} \mathrm{NMR}(100 \mathrm{MHz}$, $\left.\mathrm{CDCl}_{3}\right) \delta=199.3,196.3(\mathrm{C}=\mathrm{O}), 140.6,140.3,134.8,132.0$, 130.2, 129.8, 129.3, 129.1, 70.3, 43.1 ppm. Anal. Calcd for $\mathrm{C}_{16} \mathrm{H}_{12} \mathrm{Cl}_{2} \mathrm{O}_{3}$ (323.17): C 59.46, $\mathrm{H}$ 3.74; Found: $\mathrm{C}$ 59.40, H 3.74\%.

4-(4-Bromophenyl)-1-(4-chlorophenyl)-2-hydroxybutane-1,4-dione (5f)

White solid, m.p. $172-177^{\circ} \mathrm{C}$. FT-IR $(\mathrm{KBr}) v=3450$ $(\mathrm{O}-\mathrm{H}), 3080(\mathrm{C}-\mathrm{H}), 1674(\mathrm{C}=\mathrm{O}), 1581(\mathrm{C}=\mathrm{C}), 1197(\mathrm{C}-$ O), $771(\mathrm{C}-\mathrm{Cl}), 574(\mathrm{C}-\mathrm{Br}) \mathrm{cm}^{-1} .{ }^{1} \mathrm{H}$ NMR $(400 \mathrm{MHz}$, $\left.\mathrm{CDCl}_{3}\right) \delta=7.98\left(\mathrm{~d},{ }^{3} J_{\mathrm{H}, \mathrm{H}}=8.4 \mathrm{~Hz}, 2 \mathrm{H}, \mathrm{CH}_{\mathrm{Ar}}\right), 7.83(\mathrm{~d}$, $\left.{ }^{3} J_{\mathrm{H}, \mathrm{H}}=8.4 \mathrm{~Hz}, 2 \mathrm{H}, \mathrm{CH}_{\mathrm{Ar}}\right), 7.64\left(\mathrm{~d},{ }^{3} J_{\mathrm{H}, \mathrm{H}}=8.4 \mathrm{~Hz}, 2 \mathrm{H}\right.$, $\left.\mathrm{CH}_{\mathrm{Ar}}\right), 7.51\left(\mathrm{~d},{ }^{3} J_{\mathrm{H}, \mathrm{H}}=8.4 \mathrm{~Hz}, 2 \mathrm{H}, \mathrm{CH}_{\mathrm{Ar}}\right), 5.59-5.61(\mathrm{~m}$, $1 \mathrm{H}, \mathrm{CH}-\mathrm{O}), 4.01\left(\mathrm{~d},{ }^{3} J_{\mathrm{H}, \mathrm{H}}=6.4 \mathrm{~Hz}, 1 \mathrm{H}, \mathrm{OH}\right), 3.33-3.47$ [2×dd: $3.41-3.47\left(\mathrm{dd},{ }^{3} J_{\mathrm{H}, \mathrm{H}}=6.8,17.2 \mathrm{~Hz}, 1 \mathrm{H}, \mathrm{CH}_{2}\right)$, $\left.3.33-3.39\left(\mathrm{dd},{ }^{3} \mathrm{~J}_{\mathrm{H}, \mathrm{H}}=3.2,17.2 \mathrm{~Hz}, 1 \mathrm{H}, \mathrm{CH}_{2}\right)\right] \mathrm{ppm} .{ }^{13} \mathrm{C}$ NMR (100 MHz, $\left.\mathrm{CDCl}_{3}\right) \delta=199.3,196.6(\mathrm{C}=\mathrm{O}), 140.6$, 135.2, 132.1, 131.9, 130.2, 129.8, 129.3, 129.1, 70.3, 43.1 ppm. Anal. Calcd for $\mathrm{C}_{16} \mathrm{H}_{12} \mathrm{BrClO}_{3}$ (367.62): C 52.27, $\mathrm{H}$ 3.29; Found: C 52.30, H 3.22\%.

1-(4-Chlorophenyl)-2-hydroxy-4-(thiophen-2-yl)butane1,4-dione (5g)

Cream solid, m.p. $131.6-135.1^{\circ} \mathrm{C}$. FT-IR $(\mathrm{KBr}) v=3450$ (O-H), 3085, $2943(\mathrm{C}-\mathrm{H}), 1695(\mathrm{C}=\mathrm{O}), 1649(\mathrm{C}=\mathrm{C})$, $1203(\mathrm{C}-\mathrm{O}), 721(\mathrm{C}-\mathrm{Cl}) \mathrm{cm}^{-1} .{ }^{1} \mathrm{H} \mathrm{NMR}(400 \mathrm{MHz}$, DMSO- $\left.d_{6}\right) \delta=8.02-8.11\left(\mathrm{~m}, 4 \mathrm{H}, \mathrm{CH}_{\mathrm{Ar}}\right), 7.63\left(\mathrm{~d},{ }^{3} J_{\mathrm{H}, \mathrm{H}}=\right.$ $\left.8.4 \mathrm{~Hz}, 2 \mathrm{H}, \mathrm{CH}_{\mathrm{Ar}}\right), 7.26-7.28\left(\mathrm{~m}, 1 \mathrm{H}, \mathrm{CH}_{\mathrm{Ar}}\right), 5.92\left(\mathrm{~d},{ }^{3} J_{\mathrm{H}, \mathrm{H}}\right.$ $=7.6 \mathrm{~Hz}, 1 \mathrm{H}, \mathrm{OH}), 5.38-5.43(\mathrm{~m}, 1 \mathrm{H}, \mathrm{CH}-\mathrm{O}), 3.35-3.51$ (m, $\left.2 \mathrm{H}, \mathrm{CH}_{2}\right)$ ppm. ${ }^{13} \mathrm{C} \mathrm{NMR}\left(100 \mathrm{MHz}, \mathrm{CDCl}_{3}\right) \delta=199.2$, 190.1, 143.7, 140.5, 134.9, 133.0, 131.9, 130.3, 129.3, 128.3, 70.4, 43.9 ppm. Anal. Calcd for $\mathrm{C}_{14} \mathrm{H}_{11} \mathrm{ClO}_{3} \mathrm{~S}$ (294.75): C 57.05, H 3.76; Found: C 56.60, H 3.49\%.

1-(4-Bromophenyl)-2-hydroxy-4-phenylbutane-1,4dione (5h)

White solid, m.p. $154.5-157.0{ }^{\circ} \mathrm{C}$. FT-IR (KBr) $v=3438$ $(\mathrm{O}-\mathrm{H}), 3086(\mathrm{C}-\mathrm{H}), 1676(\mathrm{C}=\mathrm{O}), 1573(\mathrm{C}=\mathrm{C}), 1190(\mathrm{C}-$ $\mathrm{O}), 654(\mathrm{C}-\mathrm{Br}) \mathrm{cm}^{-1} .{ }^{1} \mathrm{H}$ NMR $\left(400 \mathrm{MHz}, \mathrm{DMSO}-d_{6}\right) \delta=$ 7.95-8.04 (m, 4H, CH $\left.\mathrm{CH}_{\mathrm{Ar}}\right), 7.78\left(\mathrm{~d},{ }^{3} J_{\mathrm{H}, \mathrm{H}}=8.4 \mathrm{~Hz}, 2 \mathrm{H}\right.$, $\left.\mathrm{CH}_{\mathrm{Ar}}\right), 7.67\left(\mathrm{t},{ }^{3} J_{\mathrm{H}, \mathrm{H}}=7.6 \mathrm{~Hz}, 1 \mathrm{H}, \mathrm{CH}_{\mathrm{Ar}}\right), 7.53-7.57(\mathrm{~m}, 2 \mathrm{H}$, $\left.\mathrm{CH}_{\mathrm{Ar}}\right), 5.89\left(\mathrm{~d},{ }^{3} \mathrm{~J}_{\mathrm{H}, \mathrm{H}}=7.6 \mathrm{~Hz}, 1 \mathrm{H}, \mathrm{OH}\right), 5.38-5.44(\mathrm{~m}$, $1 \mathrm{H}, \mathrm{CH}-\mathrm{O}), 3.31-3.62$ (m, 2H, $\left.\mathrm{CH}_{2}\right)$ ppm. ${ }^{13} \mathrm{C} \mathrm{NMR}(100$ MHz, DMSO- $\left.d_{6}\right) \delta=198.9,198.3(\mathrm{C}=\mathrm{O}), 136.9,134.6$, 133.9, 132.2, 131.3, 129.2, 128.5, 127.7, 69.2, 42.5 ppm. Anal. Calcd for $\mathrm{C}_{16} \mathrm{H}_{13} \mathrm{BrO}_{3}$ (333.18): C 57.68, H 3.93; Found: C 57.66, H 3.93\%.

1-(4-Bromophenyl)-4-(4-chlorophenyl)-2-hydroxybutane-1,4-dione (5i)

Cream solid, m.p. $168-173{ }^{\circ} \mathrm{C}$. FT-IR $(\mathrm{KBr}) v=3440(\mathrm{O}-$ 
H), $3074(\mathrm{C}-\mathrm{H}), 1676(\mathrm{C}=\mathrm{O}), 1558(\mathrm{C}=\mathrm{C}), 1197(\mathrm{C}-\mathrm{O})$, $771(\mathrm{C}-\mathrm{Cl}), 690(\mathrm{C}-\mathrm{Br}) \mathrm{cm}^{-1} .{ }^{1} \mathrm{H} \mathrm{NMR}\left(400 \mathrm{MHz}, \mathrm{CDCl}_{3}\right)$ $\delta=7.89-7.92\left(\mathrm{~m}, 4 \mathrm{H}, \mathrm{CH}_{\mathrm{Ar}}\right), 7.68\left(\mathrm{~d},{ }^{3} J_{\mathrm{H}, \mathrm{H}}=7.6 \mathrm{~Hz}, 2 \mathrm{H}\right.$, $\left.\mathrm{CH}_{\mathrm{Ar}}\right), 7.47\left(\mathrm{~d},{ }^{3} J_{\mathrm{H}, \mathrm{H}}=8.4 \mathrm{~Hz}, 2 \mathrm{H}, \mathrm{CH}_{\mathrm{Ar}}\right), 5.57-5.59(\mathrm{~m}$, 1H, CH-O), 4.01 (s, br., 1H, OH), 3.34-3.47 [2×dd: 3.41$3.47\left(\mathrm{dd},{ }^{3} J_{\mathrm{H}, \mathrm{H}}=7.2,17.2 \mathrm{~Hz}, 1 \mathrm{H}, \mathrm{CH}_{2}\right), 3.34-3.39(\mathrm{dd}$, $\left.\left.{ }^{3} J_{\mathrm{H}, \mathrm{H}}=3.6,17.2 \mathrm{~Hz}, 1 \mathrm{H}, \mathrm{CH}_{2}\right)\right] \mathrm{ppm} .{ }^{13} \mathrm{C} \mathrm{NMR}(100 \mathrm{MHz}$, $\left.\mathrm{CDCl}_{3}\right) \delta=199.6,196.3(\mathrm{C}=\mathrm{O}), 140.3,134.8,132.4,132.3$, 130.3, 129.8, 129.3, 129.1, 70.3, 43.1 ppm. Anal. Calcd for $\mathrm{C}_{16} \mathrm{H}_{12} \mathrm{BrClO}_{3}$ (367.62): C 52.27, H 3.29; Found: C 52.60, H 3.41\%.

1,4-Bis(4-bromophenyl)-2-hydroxybutane-1,4-dione (5j) White solid, m.p. $185-189^{\circ} \mathrm{C}$. FT-IR $(\mathrm{KBr}) v=3441$ $(\mathrm{O}-\mathrm{H}), 3081(\mathrm{C}-\mathrm{H}), 1674(\mathrm{C}=\mathrm{O}), 1575(\mathrm{C}=\mathrm{C}), 1199(\mathrm{C}-$ $\mathrm{O}), 675(\mathrm{C}-\mathrm{Br}) \mathrm{cm}^{-1} .{ }^{1} \mathrm{H}$ NMR $\left(400 \mathrm{MHz}, \mathrm{CDCl}_{3}\right) \delta=$ $7.90\left(\mathrm{~d},{ }^{3} J_{\mathrm{H}, \mathrm{H}}=8.4 \mathrm{~Hz}, 2 \mathrm{H}, \mathrm{CH}_{\mathrm{Ar}}\right), 7.83\left(\mathrm{~d},{ }^{3} J_{\mathrm{H}, \mathrm{H}}=8.0 \mathrm{~Hz}\right.$, $\left.2 \mathrm{H}, \mathrm{CH}_{\mathrm{Ar}}\right), 7.68\left(\mathrm{~d},{ }^{3} J_{\mathrm{H}, \mathrm{H}}=8.4 \mathrm{~Hz}, 2 \mathrm{H}, \mathrm{CH}_{\mathrm{Ar}}\right), 7.64(\mathrm{~d}$, $\left.{ }^{3} J_{\mathrm{H}, \mathrm{H}}=8.4 \mathrm{~Hz}, 2 \mathrm{H}, \mathrm{CH}_{\mathrm{Ar}}\right), 5.57-5.59(\mathrm{~m}, 1 \mathrm{H}, \mathrm{CH}-\mathrm{O})$, $4.00\left(\mathrm{~d},{ }^{3} J_{\mathrm{H}, \mathrm{H}}=6.0 \mathrm{~Hz}, 1 \mathrm{H}, \mathrm{OH}\right), 3.39-3.47$ [2×dd: $3.41-$ $3.47\left(\mathrm{dd},{ }^{3} \mathrm{~J}_{\mathrm{H}, \mathrm{H}}=6.8,17.2 \mathrm{~Hz}, 1 \mathrm{H}, \mathrm{CH}_{2}\right), 3.39-3.42(\mathrm{dd}$, $\left.\left.{ }^{3} J_{\mathrm{H}, \mathrm{H}}=3.6,17.2 \mathrm{~Hz}, 1 \mathrm{H}, \mathrm{CH}_{2}\right)\right] \mathrm{ppm} .{ }^{13} \mathrm{C} \mathrm{NMR}(100 \mathrm{MHz}$, $\left.\mathrm{CDCl}_{3}\right) \delta=199.5,196.5(\mathrm{C}=\mathrm{O}), 135.2,134.4,132.3$, 132.1, 130.3, 129.8, 129.3, 129.1, 70.3, 43.1 ppm. Anal. Calcd for $\mathrm{C}_{16} \mathrm{H}_{12} \mathrm{Br}_{2} \mathrm{O}_{3}$ (412.07): C 46.64, H 2.94; Found: C $46.54, \mathrm{H} 2.89 \%$.

1-(4-Bromophenyl)-2-hydroxy-4-(4-nitrophenyl)butane-

\section{1,4-dione (5k)}

Yellow solid, m.p. $146-150^{\circ} \mathrm{C}$. FT-IR (KBr) $v=3429$ (br., O-H), $3097(\mathrm{C}-\mathrm{H}), 1677(\mathrm{C}=\mathrm{O}), 1581(\mathrm{C}=\mathrm{C}), 1519$, $1328\left(\mathrm{NO}_{2}\right), 1195(\mathrm{C}-\mathrm{O}), 688(\mathrm{C}-\mathrm{Br}) \mathrm{cm}^{-1} .{ }^{1} \mathrm{H}$ NMR $(400$ MHz, DMSO- $\left.d_{6}\right) \delta=8.36\left(\mathrm{~d},{ }^{3} J_{\mathrm{H}, \mathrm{H}}=8.8 \mathrm{~Hz}, 2 \mathrm{H}, \mathrm{CH}_{\mathrm{Ar}}\right)$, $8.22\left(\mathrm{~d},{ }^{3} \mathrm{~J}_{\mathrm{H}, \mathrm{H}}=8.8 \mathrm{~Hz}, 2 \mathrm{H}, \mathrm{CH}_{\mathrm{Ar}}\right), 7.98\left(\mathrm{~d},{ }^{3} \mathrm{~J}_{\mathrm{H}, \mathrm{H}}=8.4 \mathrm{~Hz}\right.$, $\left.2 \mathrm{H}, \mathrm{CH}_{\mathrm{Ar}}\right), 7.78\left(\mathrm{~d},{ }^{3} J_{\mathrm{H}, \mathrm{H}}=8.4 \mathrm{~Hz}, 2 \mathrm{H}, \mathrm{CH}_{\mathrm{Ar}}\right), 6.00(\mathrm{~d}$, $\left.{ }^{3} J_{\mathrm{H}, \mathrm{H}}=7.6 \mathrm{~Hz}, 1 \mathrm{H}, \mathrm{OH}\right), 5.41-5.46(\mathrm{~m}, 1 \mathrm{H}, \mathrm{CH}-\mathrm{O})$, $3.44-3.69$ [2×dd: $3.62-3.69\left(\mathrm{dd},{ }^{3} J_{\mathrm{H}, \mathrm{H}}=6.4,17.2 \mathrm{~Hz}, 1 \mathrm{H}\right.$, $\left.\left.\mathrm{CH}_{2}\right), 3.44-3.49\left(\mathrm{dd},{ }^{3} \mathrm{~J}_{\mathrm{H}, \mathrm{H}}=6.0,17.2 \mathrm{~Hz}, 1 \mathrm{H}, \mathrm{CH}_{2}\right)\right] \mathrm{ppm}$. ${ }^{13} \mathrm{C}$ NMR (100 MHz, DMSO- $\left.d_{6}\right) \delta=199.2,196.2(\mathrm{C}=\mathrm{O})$, 151.1, 140.9, 139.6, 132.4, 130.3, 129.5, 129.4, 124.0, 70.3, 43.6 ppm. Anal. Calcd for $\mathrm{C}_{16} \mathrm{H}_{12} \mathrm{BrNO}_{5}$ (378.17): C 50.82, H 3.20, N 3.70; Found: C 50.80, H 3.21, N 3.89\%.

\section{1-(4-Bromophenyl)-4-(furan-2-yl)-2-hydroxybutane-}

\section{1,4-dione (5l)}

Cream solid, m.p. $168.0-173.0^{\circ} \mathrm{C}$. FT-IR $(\mathrm{KBr}) \nu=3620$ $(\mathrm{O}-\mathrm{H}), 1679(\mathrm{C}-\mathrm{O}), 1550(\mathrm{C}=\mathrm{C}), 1198(\mathrm{C}=\mathrm{O}), 675(\mathrm{C}-\mathrm{Br})$ $\mathrm{cm}^{-1} .{ }^{1} \mathrm{H}$ NMR (400 MHz, DMSO- $\left.d_{6}\right) \delta=7.96\left(\mathrm{~d},{ }^{3} J_{\mathrm{H}, \mathrm{H}}=\right.$ $\left.8.4 \mathrm{~Hz}, 2 \mathrm{H}, \mathrm{CH}_{\mathrm{Ar}}\right), 7.92\left(\mathrm{~d},{ }^{3} \mathrm{~J}_{\mathrm{H}, \mathrm{H}}=8.4 \mathrm{~Hz}, 2 \mathrm{H}, \mathrm{CH}_{\mathrm{Ar}}\right)$, 7.75-7.79 (m, 3H, $\left.\mathrm{CH}_{\mathrm{Ar}}\right), 5.91\left(\mathrm{~d},{ }^{3} \mathrm{~J}_{\mathrm{H}, \mathrm{H}}=7.6 \mathrm{~Hz}, 1 \mathrm{H}, \mathrm{OH}\right)$, 5.37-5.42 (m, 1H, CH-O), 3.33-3.59 (m, 2H, $\left.\mathrm{CH}_{2}\right) \mathrm{ppm}$. ${ }^{13} \mathrm{C}$ NMR (100 MHz, DMSO- $\left.d_{6}\right) \delta=198.8,197.6(\mathrm{C}=\mathrm{O})$,
135.9, 134.6, 132.3, 132.2, 131.3, 130.6, 127.9, 127.8, 69.4, 42.5 ppm. Anal. Calcd for $\mathrm{C}_{14} \mathrm{H}_{11} \mathrm{BrO}_{4}$ (323.14): $\mathrm{C}$ 52.04, H 3.43; Found: C 52.20, H 3.40\%.

Acknowledgments. This work was supported by the research council of the PNU and UM. B. E. S. gratefully acknowledges Mr. Biglari (IASBS) for taking NMR spectra. And the publication cost of this paper was supported by the Korean Chemical Society.

\section{REFERENCES}

1. (a) Anastas, P.; Williamson, T. Green Chemistry, Frontiers in Benign Chemical Synthesis and Procedures; Oxford Science Publications: New York, 1998. (b) Hayashi, T.; Yamasaki, K. Chem. Rev. 2003, 103, 2829.

2. (a) Grieco, P. A. Organic Synthesis in Water; Blackie Academic and Professional: London, 1998. (b) Li, C.-J. Chem. Rev. 2005, 105, 3095. (c) Li, C.-J.; Chan, T.-H. Organic Reactions in Aqueous Media; Wiley: New York, 1997. (d) Zuo, H.; Li, Z.-B.; Zhao, B.-X.; Miao, J.-Y.; Meng, L.-J.; Jang, K.; Ahn, C.; Lee, D.-H.; Shin, D.-S. Bull. Korean Chem. Soc. 2011, 32, 2965. (e) Islam, M.; Hossain, D.; Mondal, P.; Roy, A. S.; Mondal, S.; Mobarak, M. Bull. Korean Chem. Soc. 2010, 31, 3765. (f) Dhokte, A. O.; Khillare, S. L.; Lande, M. K.; Arbad, B. R. J. Korean Chem. Soc. 2011, 55, 430.

3. (a) Marhwald, R. Modern Aldol Reactions; Weinheim Wiley-VCH: 2004. (b) Trost, B. M. Science 1991, 254, 1471. (c) Trost, B. M. Angew. Chem., Int. Ed. 1995, 34, 259. (d) Li, J.-Z.; Cui, B.-C.; Wang, J. J.; Shim, Y. K. Bull. Korean Chem. Soc. 2011, 32, 2465. (e) Basaif, S. A.; Sobahi, T. R.; Khalil, A. K.; Hassan, M. A. Bull. Korean Chem. Soc. 2005, 26, 1677. (f) Jeong, T. M.; Park, K. H. J. Korean Chem. Soc. 1989, 33, 426.

4. (a) Scheidt, K. A.; Bannister, T. D.; Tasaka, A.; Wendt, M. D.; Savall, B. M.; Fegley, G. J.; Roush, W. R. J. Am. Chem. Soc. 2002, 124, 6981. (b) White, J. D.; Blakemore, P. R.; Green, N. J.; Hauser, E. B.; Holoboski, M. A.; Keown, L. E.; Kolz, C. S. N.; Phillips, B. W. J. Org. Chem. 2002, 67, 7750. (c) Gerber-Lemaire, S.; Vogel, P. Eur. J. Org. Chem. 2003, 2959. (d) Casiraghi, G.; Zanardi, F.; Appendino, G.; Rassu, G. Chem. Rev. 2000, 100, 1929.

5. (a) Nicolaou, K. C.; Vourloumis, D.; Wissinger, N.; Baran, P. S. Angew. Chem., Int. Ed. 2000, 39, 44. (b) Evans, D. A.; Ratz, A. M.; Huff, B. E.; Sheppard, G. S. J. Am. Chem. Soc. 1995, 117, 3448. (c) Keck, G. E.; Wager, C. A.; Sell, T.; Wager, T. T. J. Org. Chem. 1999, 64, 2172.

6. (a) Stetter, H.; Kulhmann, H. Synthesis 1975, 379. (b) Mussatto, M. C.; Savoia, D.; Trombini, C.; Umani-Ronchi, A. J. Org. Chem. 1980, 45, 4002. (c) Wenkert, E.; Greenberg, R. S.; Ragu, M. S. J. Org. Chem. 1985, 50, 4681. (d) Takahashi, K.; Aihara, T.; Ogura, K. Chem. Lett. 1987, 2359. (e) Curran, D. P. Tetrahedron 1994, 58, 1181. (f) Banik, 
B. K.; Banik, I.; Renteria, M.; Dasgupta, S. K. Tetrahedron Lett. 2005, 46, 2643. (g) Ho, T.-L. Synth. Commun. 1974, 4, 265. (h) Ballini, R.; Petrini, M. Synth. Commun. 1989, 19, 575. (i) Yamashita, M.; Tashika, H.; Uchida, M. Bull. Chem. Soc. Jpn. 1992, 65, 1257. (j) Bentley, P. H. Chem. Soc. Rev. 1974, 29. (k) Mitra, A. The Synthesis of Prostaglandins; Wiley: New York, 1977. (l) Bindra, J. S.; Bindra, R. Prostaglandin Synthesis; Academic Press: New York, 1977.

7. (a) Gribble, G. W. In Comprehensive Heterocyclic Chemistry; Katrizky, A. R., Rees, C. W., Scriven, E. F., Eds.; Pergamon: Oxford, 1996; Vol. 2, p. 207. (b) Benassi, R. In Comprehensive Heterocyclic Chemistry; Katrizky, A. R., Rees, C. W., Scriven, E. F., Eds.; Pergamon: New York, 1996; Vol. 2, p. 259. (c) Hirano, K.; Biju, A. T.; Piel, I.; Glorius, F. J. Am. Chem. Soc. 2009, 131, 14190. (d) Biju, A. T.; Glorius, F. Angew. Chem., Int. Ed. 2010, 49, 9761. (e) Mortensen, D. S.; Rodriguez, A. L.; Carlson, K. E.; Sun, J.; Katzenellenbogen, B. S.; Katzenellenbogen, J. A. J. Med. Chem. 2001, 44, 3838. (f) El-Haji, T.; Martin, J. C.; Descotes, G. J. J. Heterocycl. Chem. 1983, 20, 233. (g) Perrine, D. M.; Kagan, J.; Huang, D. B.; Zeng, K.; Theo, B. K. J. Org. Chem. 1987, 52, 2213. (h) Bellina, F.; Rossi, R. Tetrahedron 2006, 62, 7213. (i) Lipshutz, B. H. Chem. Rev. 1986, 86, 795. (j) Rao, H. S. P.; Jothilingam, S. J. Org. Chem. 2003, 68, 5392.

8. (a) Katritzky, A. R.; Zhang, G. F.; Jiang, J. L. J. Org. Chem. 1995, 60, 7605. (b) Lu, X. Y.; Ji, J. G.; Ma, D. W.; Shen, W. J. Org. Chem. 1991, 56, 5774. (c) Yasuda, M.; Tsuji, S.; Shigeyoshi, Y.; Baba, A. J. Am. Chem. Soc. 2002, 124, 7440. (d) Yuguchi, M.; Tokuda, M.; Orito, K. J. Org. Chem. 2004, 69, 908. (e) Xue, A. S.; Li, L.; Liu, Y.; Guo, Q. J. Org. Chem. 2006, 71, 215. (f) Matsubara, R.; Doko, T.; Uetake, R.; Kobayashi, S. Angew. Chem., Int. Ed. 2007, 46, 3047

9. Seyferth, D.; Hui, R. C. J. Am. Chem. Soc. 1985, 107, 4551.

10. (a) Cooke, M. P., Jr.; Parlman, R. M. J. Am. Chem. Soc. 1977, 99, 5222. (b) Hegedus, L. S.; Perry, R. J. J. Org. Chem. 1985, 50, 4955. (c) Hermanson, J. R.; Hershberger, J. W.; Pinhas, A. R. Organometallics 1995, 14, 5426. (d) Lipshutz, B. H.; Elworthy, T. R. Tetrahedron Lett. 1990, $31,477$.
11. (a) Hassner, A.; Lokanatha Rai, K. M. In Comprehensive Organic Synthesis; Trost, B. M., Fleming, I., Eds.; Pergamon: Oxford, 1991; Vol. 2, p 541. (b) Stetter, H.; Kuhlmann, H. Org. React. 1991, 40, 407. (c) Mattson, A. E.; Bharadwaj, A. R.; Scheidt, K. A. J. Am. Chem. Soc. 2004, 126, 2314. (d) Johnson, J. S. Angew. Chem., Int. Ed. 2004, 43, 1326. (e) Myers, M. C.; Bharadwaj, A. R.; Milgram, B. C.; Scheidt, K. A. J. Am. Chem. Soc. 2005, 127, 14675.

12. Calter, M. A.; Zhu, C. Org. Lett. 2002, 4, 205.

13. Enders, D.; Balensiefer, T. Acc. Chem. Res. 2004, 37, 534.

14. (a) Stetter, H.; Kuhlmann, H. Synthesis 1975, 379. (b) Yadav, J. S.; Anuradha, K.; Reddy, B. V. S.; Eeshwaraiah, B. Tetrahedron Lett. 2003, 44, 8959. (c) Amarnath, V.; Anthony, D. C.; Amarnath, K.; Valentine, W. M.; Wetterau, L. A.; Graham, D. G. J. Org. Chem. 1991, 56, 6924. (d) Kerr, M. S.; Read de Alaniz, J.; Rovis, T. J. Org. Chem. 2005, 70, 5725.

15. Smith, A. B., III; Adams, C. M. Acc. Chem. Res. 2004, 37,365

16. Eftekhari-Sis, B.; Zirak, M.; Akbari, A. Chem. Rev. 2013, doi: $10.1021 / \mathrm{cr} 300176 \mathrm{~g}$.

17. Tokuda, O.; Kano, T.; Gao, W.-G.; Ikemoto, T.; Maruoka, K. Org. Lett. 2005, 7, 5103.

18. (a) Allen, C. F. H. Can. J. Res. 1931, 4, 264. (b) Allen, C. F. H.; Van Allan, J. A. J. Org. Chem. 1951, 16, 716. (c) Hara, N.; Nakamura, S.; Shibata, N.; Toru, T. Adv. Synth. Catal. 2010, 352, 1621. (d) Samanta, S.; Zhao, C.-G. Tetrahedron Lett. 2006, 47, 3383. (e) Langer, P.; Köhler, V. Org. Lett. 2000, 2, 1597.

19. Zhao, J.; Zheng, K.; Yang, Y.; Shi, J.; Lin, L.; Liu, X.; Feng, X. Synlett 2011, 903.

20. Riley, H. A.; Gray, A. R. Organic Syntheses, Wiley \& Sons: New York, 1943, collect; Vol. II, p. 509.

21. By permission of Allgood, T. Scientific Account Manager CCDC; Macrae, C. F.; Edgington, P. R.; McCabe, P.; Pidcock, E.; Shields, G. P.; Taylor, R.; Towler, M.; van de Streek, J. J. Appl. Cryst. 2006, 39, 453.

22. Eftekhari-Sis, B.; Akbari, A.; Amirabedi, M. Chem. Heterocycl. Comp. 2011, 46, 1330.

23. Harms, K. Private communication to the Cambridge Structural Database. deposition number CCDC 784184. 